\title{
INNOVATIONS IN VACCINE DEVELOPMENT: WHAT NEXT?
}

\section{Dear Reader,}

COVID-19 pandemic has affected the whole world in a way that is unprecedented. The origin of the SARS-CoV-2 and the true scale of number of cases and deaths in every country is debatable. Nevertheless, it is clear that the spread of the virus is continuing worldwide with a devastating effect on the global economy. The control of the pandemic was essentially by implementing strict measures such as social distancing, use of personal protective equipment, personal hygiene and sanitation. There were numerous occasions since the beginning of the pandemic when governments across the world had rejoiced that their measures to control the spread of the infections had worked. However, non-compliance of safety measures and new strains of the virus led to the rise of the next wave of infections.

SARS-CoV-2 gene sequence was published in the beginning of the year 2020 and since then researchers, both academic and industrial, have worked hard in the development of a range of vaccines. The list of new vaccines include viral vector vaccines (AstraZeneca/Oxford/Serum Institute India; Jannsen; CanSino; Gamaleya), mRNA based vaccines (Pfizer/BioNTech; Moderna; CureVac; Inovio Pharmaceuticals) and inactivated vaccines (Bharat Biotech; Sinovac; Sinopharm), to name a few. Most of these vaccines were approved for human use by the regulatory bodies. There are a number of other vaccines, including attenuated vaccines and protein vaccines that are currently in various stages of clinical trials around the world.

India is the largest vaccine supplier with Serum Institute India being the single largest vaccine manufacturer in the world. In January 2021, India was the largest producer and supplier of COVID-19 vaccines to the world. Millions of doses have been produced and supplied free to countries in need as a goodwill gesture too. Yet, India mostly depends on other countries, especially USA and EU, for the supply of consumables for vaccine production. According to a report by the world trade organisation, a typical vaccine production may use thousands of different materials that are sourced from suppliers around the world. Some of these essential materials for producing a simple vaccine would include cell culture media, a bioreactor bag, filters for clarification/purification/sterilisation and micro-carrier beads for protein growth to name a few. The manufacturer prepares and submits to the regulatory body a master document containing details of the raw materials, suppliers, the production process, as well as details of clinical trials for their approval before the vaccine can be manufactured for general supply and use.

Political decisions made by the former government of USA introduced various restrictions on the supply of vaccine consumables. These decisions were possibly made to ensure that the local demands were met before any of the resources can be exported. This led to a serious shortage in India of raw materials, packaging materials, critical consumables such as culture media and various equipment due to these embargoes. These restrictions have hit the production of vaccines for majority of the suppliers around the world. In such a situation, can the vaccine manufacturers approach suppliers in other countries such as in China or Russia? Unfortunately, this is not quite so simple. It would be difficult to make any changes to the raw materials or suppliers for producing an approved vaccine and it would require a thorough validation as well as applying for regulatory clearance, again. This would lead to severe delays in the production and immunisation of the general population, with an obvious increase to the costs involved.

How would a change of supplier affect vaccine production? Any change in raw material has the potential for the quality of the product being affected. The main issue is with maintaining quality and safety. Although US is not the sole supplier of these consumables with other countries like China and Russia having some capacity, it does play a predominant role in vaccine manufacture. There were reports of some batches of 
Johnson and Johnson and AstraZeneca vaccines manufactured by a US based company rejected by the FDA for failing manufacturing quality. A key principle that is followed by pharmaceutical/biopharmaceutical manufacturers around the world is, "do no harm".

As mentioned previously, some of the essential consumables in vaccine production such as cell culture media, bioreactor bags, filters and micro-carrier beads are predominantly manufactured and exported by biotechnology industries based in the USA. For example, cell culture media is produced by HyClone Laboratories Inc. and Merck Millipore (both based in USA), Lonza Group AG (Switzerland), CellGenix Gmbh (Germany) and HiMedia Laboratories (India). The cell culture media market share is valued at $\$ 4.65 \mathrm{~b}$ USD in 2020 and is projected to increase to $\$ 8.24 \mathrm{~b}$ USD by 2028, a compound annual growth rate (CAGR) of 7.38\%. Similarly, the expected CAGR by 2027 for other consumables such as single use bioreactor systems is $15.5 \%$ (\$6.39b USD), for micro carrier beads to be $5.52 \%$ ( $\$ 1.9 b$ USD) and for micro filters is expected to be $7.7 \%$ ( $\$ 8.48 b$ USD).

On a positive note, the new technologies that were developed to tackle SARS-CoV-2 pandemic have relied on the quality and procurement of consumables from suppliers around the world. These vaccines were developed, tested and implemented in less than a year. These are extraordinary medical and scientific breakthroughs. The pandemic is ongoing and it is vital that the current immunisation drive goes through unhindered. There is a fair prospect that these developments will help in controlling this and any future pandemic. The ultimate goal is to produce the vaccines in an environment that is independent of unexpected supply chain interruptions.

Therefore, there is a unique opportunity for Indian Pharmaceutical, Biotechnological and Engineering firms to look at the production of not only the finished products such as vaccines but also to manufacture the essential raw materials. This would not only increase the self-reliance of Indian biotechnology industry but will also help in controlling the price of the vaccines produced. However, the major challenge for this approach is satisfying the quality and regulatory requirements of the raw materials to meet the stringent specifications for vaccine manufacture.

Dr Ananth Pannala, MPharm,

PhD, FRSC, SFHEA

https://doi.org/10.53879/id.58.06.p0005

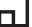

\section{About The Guest Editor}

Dr Pannala is a Principal Lecturer in Pharmaceutics in the School of Pharmacy and Biomolecular Sciences, University of Brighton, UK. His research interest and expertise includes development and evaluation of new technologies based on nanotechnology and microtechnology for delivering therapeutically active molecules for targeted drug delivery.

Dr Pannala completed his BPharm and MPharm from College of Pharmaceutical Sciences, Manipal and his PhD from King's College London. He is a Fellow of the Royal Society of Chemistry and a Senior Fellow of Higher Education Academy. Dr Pannala has nearly 30 years of research experience in both academia and pharmaceutical industry.

Dr Pannala has more 30 publications in peer-reviewed international journals, which have been cited more than 25,000 times. He is the Lead for the Biomaterials and Drug Delivery Research and Enterprise group at University of Brighton. He has so far guided $7 \mathrm{PhD}$ students as a principal supervisor and more than 75 Masters Students in their research projects. He has been successful, as a co-applicant, in procuring research income from various funding bodies. Dr Pannala is a reviewer of grant applications for RCUK, British Council, Horizon 2020 and others. He regularly reviews journal articles for international research journals in the field of Pharmaceutics, Pharmacology and Biomedical Sciences.

म

If you would like to comment on the editorial please write to us at publications@idmaindia.com 